\title{
Designing Clinical Space for the Delivery of Integrated Behavioral Health and Primary Care
}

\author{
Rose Gunn, MA, Melinda M. Davis, PhD, Jennifer Hall, MPH, John Heintzman, MD, \\ John Muench, MD, MPH, Brianna Smeds, AIA, Benjamin F. Miller, PsyD, \\ William L. Miller, MD, MA, Emma Gilchrist, MPH, Shandra Brown Levey, PhD, \\ Jacqueline Brown, MSN, APN, Pam Wise Romero, PhD, and Deborah J. Cohen, PhD
}

Purpose: This study sought to describe features of the physical space in which practices integrating primary care and behavioral health care work and to identify the arrangements that enable integration of care.

Methods: We conducted an observational study of 19 diverse practices located across the United States. Practice-level data included field notes from 2-4-day site visits, transcripts from semistructured interviews with clinicians and clinical staff, online implementation diary posts, and facility photographs. A multidisciplinary team used a 4-stage, systematic approach to analyze data and identify how physical layout enabled the work of integrated care teams.

Results: Two dominant spatial layouts emerged across practices: type-1 layouts were characterized by having primary care clinicians (PCCs) and behavioral health clinicians (BHCs) located in separate work areas, and type-2 layouts had BHCs and PCCs sharing work space. We describe these layouts and the influence they have on situational awareness, interprofessional "bumpability," and opportunities for on-the-fly communication. We observed BHCs and PCCs engaging in more face-to-face methods for coordinating integrated care for patients in type 2 layouts $(41.5 \%$ of observed encounters vs $11.7 \% ; P<$ $.05)$. We show that practices needed to strike a balance between professional proximity and private work areas to accomplish job tasks. Private workspace was needed for focused work, to see patients, and for consults between clinicians and clinical staff. We describe the ways practices modified and built new space and provide 2 recommended layouts for practices integrating care based on study findings.

Conclusion: Physical layout and positioning of professionals' workspace is an important consideration in practices implementing integrated care. Clinicians, researchers, and health-care administrators are encouraged to consider the role of professional proximity and private working space when creating new facilities or redesigning existing space to foster delivery of integrated behavioral health and primary care. (J Am Board Fam Med 2015;28:S52-S62.)

Keywords: Behavioral Medicine; Delivery of Health Care, Integrated; Medical Office Buildings; Primary Health Care; Qualitative Research

Layout of physical space affects how professionals work together across diverse settings. ${ }^{1-4}$ In health care, design of the physical environment has been

This article was externally peer reviewed.

Submitted 8 February 2015; revised 15 May 2015; accepted 22 May 2015.

From the Department of Family Medicine, Oregon Health \& Science University, Portland (R.G., M.D., J.Ha., J.He., J.M., D.J.C.); Oregon Rural Practice-Based Research Network, Oregon Health \& Science University, Portland (M.D.); PKA Architects, P.C., Portland, OR (M.D., B.S.); Department of Family Medicine, University of Colorado School of Medicine, Aurora (B.F.M., E.G., S.B.L.); Department of Family Medicine, Lehigh Valley Health Network, Allentown, PA (W.L.M.); Southeast Mental Health Group, associated with health-care quality, work efficiency, and cost effectiveness. ${ }^{5,6}$ However, most research on evidence-based health design has focused on

La Junta, CO (J.B.); Axis Health Systems, Durango, CO (P.W.R.); Department of Medical Informatics and Clinical Epidemiology, Oregon Health \&; Science University, Portland (D.J.C.)

Funding: This work is funded by grants from The Colorado Health Foundation (CHF-3848), Agency for Healthcare Research and Quality (8846.01-S01), Tides Foundation/CalMHSA Integrated Behavioral Health Project (AWD-131237), and Maine Health Access Foundation (2012FI-0009). MD's time is partially supported by an Agency for Healthcare Research \& Quality-funded PCOR K12 award (Award No. 1 K12 HS022981 01). 
hospital settings, and design principles for primary care or community-based mental health settings are limited. ${ }^{5-8}$ A 2009 review suggested that physical features of outpatient settings are associated with patient outcomes; however, the literature described focused on older models of "physician-centered" primary care rather than new, collaborative, team-based approaches. ${ }^{5}$

Optimizing the design and layout of physical space is an important consideration for practices implementing new, team-based approaches to care. ${ }^{3}$ Increasingly, practices nationally and internationally are integrating mental health and physical services to improve quality, experience, and coordination of care for people with complex or co-occurring conditions. ${ }^{9,10}$ However, few studies have examined how practice layout or professional proximity fosters or impairs coordination among professionals in practices integrating care. To date, research in this area suggests that merely placing professionals in the same building does not enable team-based care. ${ }^{9}$ Proximity of professionals working in the same location shapes professionals' feelings of isolation ${ }^{10}$ as well as rates of engaging other professionals in patient care. ${ }^{11}$

Therefore, the purpose of this study was to describe the observed spatial designs in 19 diverse practices integrating primary care and behavioral health and to identify how physical space and clinician proximity was connected to the behaviors of PCCs and BHCs working on these teams. Based on this analysis, we offer design recommendations that can inform practices as they develop and adapt their settings to deliver integrated care.

\section{Methods}

This was an observational study that used qualitative data to draw comparisons between 19 diverse practice sites. The Institutional Review Boards at Oregon Health \& Science University and the University of Texas approved this study.

\section{Sample}

We examined 19 primary care and community mental health centers in the United States. Eleven

Conflict of interest: none declared.

Corresponding author: Rose Gunn, MA, Department of Family Medicine, Oregon Health \& Science University, 3181 SW Sam Jackson Park Rd, Mail Code: FM, Portland, OR 97239 (Email: gunnr@ohsu.edu). practices from Colorado were selected by a steering committee to participate in the Advancing Care Together (ACT) program. ${ }^{12}$ Eight practices were selected by an expert panel to participate in an Integration Workforce Study (IWS). These practices were located across the United States and selected for their experience integrating care. ${ }^{13}$

\section{Data Collection}

The same multidisciplinary research team collected data from both ACT and IWS practices. Two to 4 experienced researchers conducted site visits at each practice, which were 2 to 4 days long, depending on practice size. We focused on intensively observing all aspects of clinical operations, including teamwork areas and encounters with patients in and out of examination and visit rooms. We shadowed primary care and BHCs, and joined their visits when patients permitted. We took photographs of the external facility and inside the practice, collected floor plans, and conducted semistructured interviews with practice members who represented diverse clinical roles (eg, administrator, office manager, BHCs, PCCs, medical assistant). Clinic members from each ACT site also routinely posted in online implementation diaries. ${ }^{14}$

\section{Data Management}

Interviews were audio recorded and professionally transcribed. Transcripts were checked for accuracy. During the site visit, the research team took notes or jottings that were written up within 24 hours as detailed field notes. Photographs, diary posts, field notes, and interview transcripts were deidentified and entered into Atlas.ti (Version 7.0, Atlas.ti Scientific Software Development, GmbH) for data management and analysis.

\section{Analysis}

A multi-disciplinary team composed of an anthropologist, social and clinical psychologists, communication scientist, public health specialist, primary care physicians, and architect analyzed data in 4 steps. First, we examined the photographs from each practice while reading field notes and interview data. This allowed us to develop an understanding of each practice and to get a sense of the physical layout of the practice and how clinical teams used these spaces to deliver integrated care. Second, we examined findings across practices to identify common patterns regarding the use of 
physical space. Through this process we identified 2 common practice layouts based on BHC and PCC proximity. Within each of these layouts, we examined how integrated-care team members worked together in the space to deliver care, making comparisons between the 2 layouts we identified. We felt that it might be useful to express what we were observing in our qualitative findings numerically, and our third step was to count the times we observed PCCs and BHCs working together face to face to coordinate and integrate care for patients. We used a Z-test to compare whether the proportion of patient encounters in which BHCs and PCCs were observed coordinating care differed significantly between the 2 different practice layouts. Fourth, we shared preliminary findings from this analysis with an architect specializing in health care design, who provided additional insights into the design features we were observing, helped identify design strategies that could be employed by practices who were remodeling or building new space to deliver integrated care, and drafted figures for 2 floor plans that might support integrated care teams based on our observations.

\section{Results}

Practices varied in size, ownership, location, and integration approach. ${ }^{12,13}$ Three practices built new facilities with plans for delivering integrated care. Three practices remodeled their facilities and 5 repurposed existing space to accommodate new professionals (eg, shifting office locations so that clinicians from different backgrounds were in close proximity; placing BHCs and PCCs in the same office). Table 1 summarizes practice characteristics, the layout of each practice, and characteristics of practices' approach to integration.

\section{Two Observed Layouts: Separate Work Areas (Type 1) or Shared Work Areas (Type 2)}

Two general layouts of physical space emerged across the 19 practices based on BHC and PCC provider proximity; example layouts are shown in Figures 1 and 2. Layout type 1 (Figure 1) was characterized by separate, private offices for PCCs and BHCs. Clinician offices could be located on the same floor or on separate floors in practices with type-1 layouts. Frequently, the office of the clinician who was "added" to the practice (eg, BHC, PCC) was located in an area remote from the work- flow of routine patient care. New clinicians working in practices with type-1 layouts often reported feeling isolated from other members of the care team. In Practice 18, a physician's assistant (PA) was added to a community mental health center. The PA's examination room was distant from where patients checked in, had to be accessed through a locked corridor, and was removed from the offices used by other members of the care team (ie, health care coordinator, substance use counselor, medical assistant). The PA reported feeling isolated with this arrangement:

(The) small examination room and lab-
oratory area are far removed from
where the medical assistant checks in
patients and [the Health Care Coordi-
nator] and [Substance Use Counselor]
meet with patients. I feel isolated from
the team at times. (Diary entry from
PA, Practice 18)

In contrast, practices with type-2 layouts were characterized by shared office space for PCCs and BHCs, either by locating professionals within the same room or clustering offices into smaller groups or "pods" for care delivery. Practices displayed many variations on this design, from solo practices where the PCC and BHC shared a private office, to large practices where 4 clinical teams were clustered into pods within the same open office space. The excerpt below describes one of these variations:

The practice area is open. Each team sits together in a small, open work area with desks. There are some dividers between teams that go up high enough that there is a slight sound barrier. It is minimal. You can hear and see across them, and within a team there are low dividers, if any. You can see and talk to folks easily because they are sitting right next to you, and you are looking right at them. In the team area the doctor, $\mathrm{RN}$ [registered nurse] case manager, case manager support, scheduler, and medical assistant are all sitting together. The $\mathrm{BHC}$ sits near the team. Since there are 4 teams and 2 BHCs, they sit really close to 1 team and just a few feet from the other team. (Field Notes, Practice 3) 


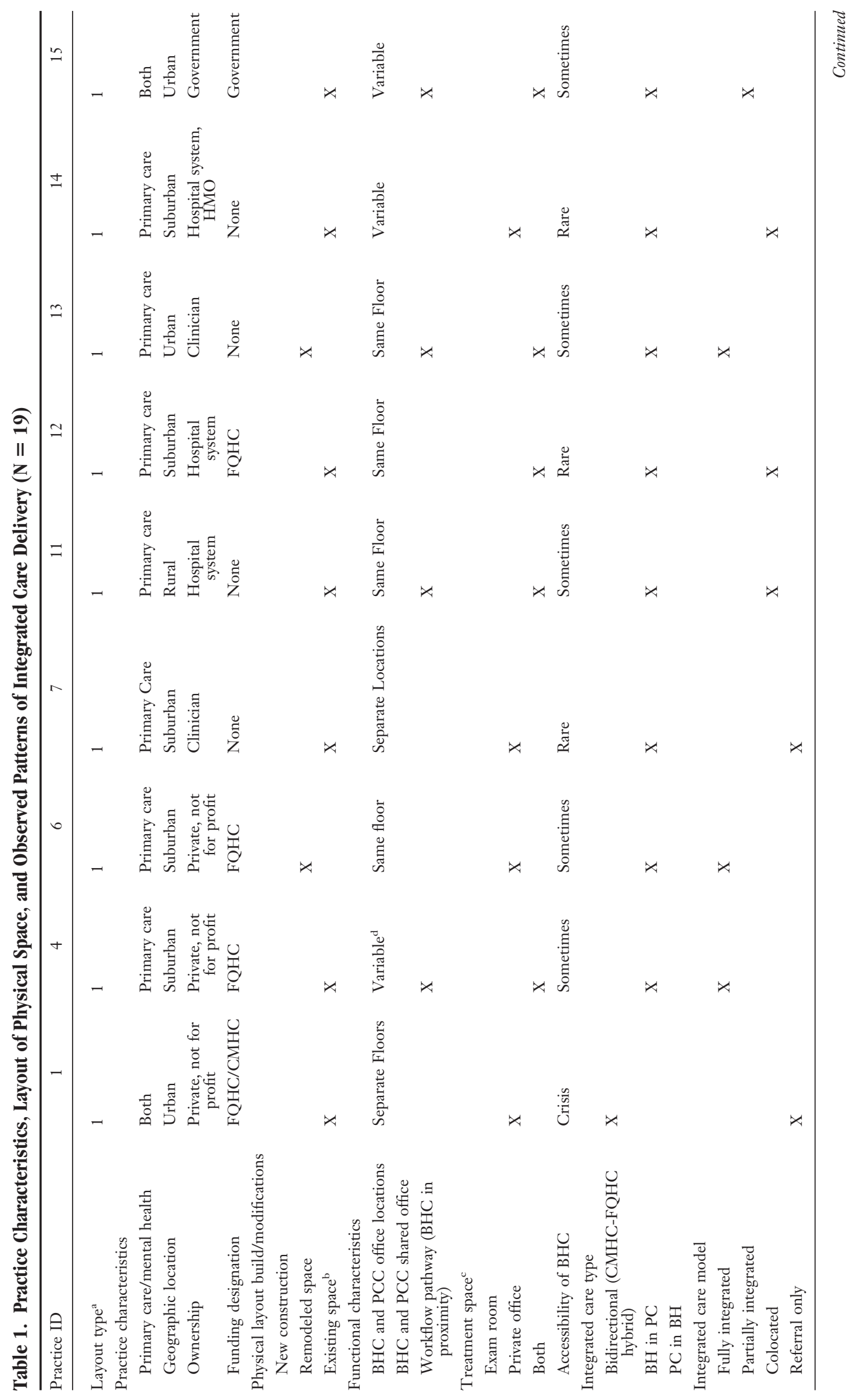




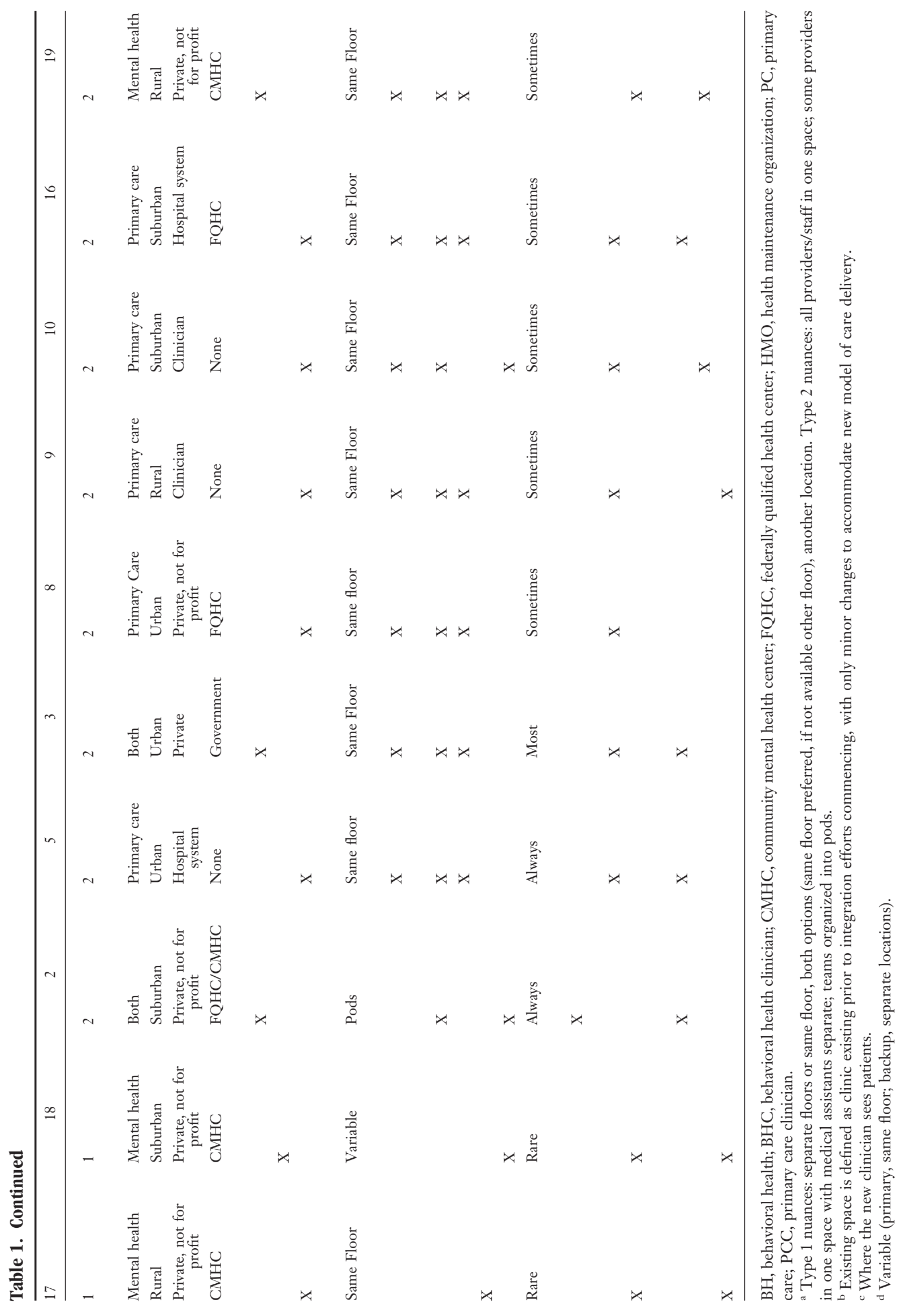


Figure 1. Observed type 1 layout: primary care and behavioral health clinicians located in separate work areas.
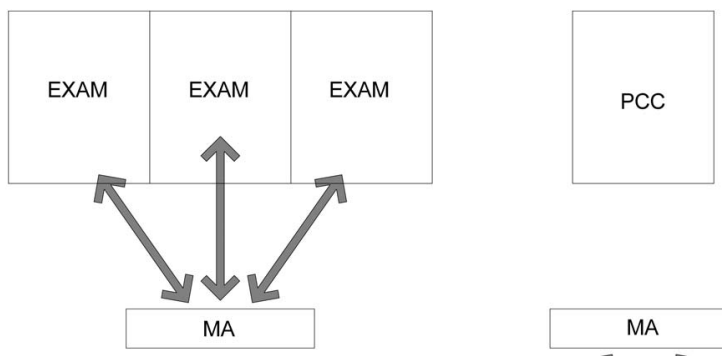

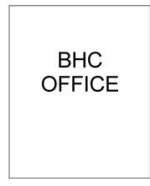

Although shared offices ranged in size, they included professional workspaces (eg, desk, computer) arranged in close proximity, so team members worked shoulder to shoulder. Shared offices had open space designs that afforded little privacy but facilitated opportunity for cross talk among professionals. Other team members, such as medical assistants, nurses, or pharmacists, could be located in the same office, depending on size and availability of space, or in workspaces nearby.

\section{Two Key Dimensions of Physical Layout: Proximity and Private Work Space}

We observed 2 key dimensions of physical layout that were in tension and needed to be balanced in practices working to integrate care: the proximity of BHCs and PCCs and the presence of workspace that enabled professionals to perform job duties that required privacy. We show how different ways of working together to coordinate patient care emerged in these differing spatial arrangements.

\section{BHC and PCC Provider Proximity}

Physically locating BHC and PCCs in close proximity had implications for real-time communication. Practice 5, an urban primary care clinic serving complex patients, had a type-2 layout where BHCs and PCCs shared a small office space. Shared workspace enabled clinicians to routinely discussed patient care plans when a need emerged, as illustrated in the following excerpt:

The doctor is reviewing the chart of the patient she is seeing first thing this morning. She leans over to BHC who works in the same office space and says the patient's name. She says that you have seen her a couple of times. It says

Figure 2. Observed type 2 layout: primary care and behavioral health clinicians located in shared work areas.

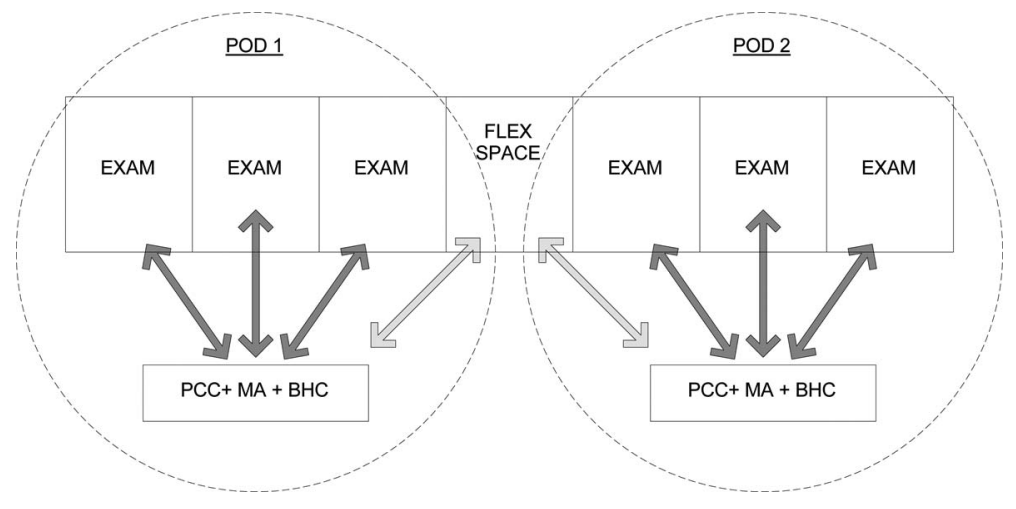


that the patient is here for followup pain. The doctor asks whether the patient needs to have a behavioral health visit. The BHC says that this patient is floating back and forth between here and another city. She has kids scattered around. Her son is getting out of treatment and so she wants to be here to support him. The BHC says it is probably a good idea for me to pop in and meet with her. (Field Notes, Practice 5)

As this example demonstrates, professionals working in close proximity could lean over and talk with each other about a range of care issues. In addition, close proximity allowed teams to establish situational awareness, an understanding of the tasks in which other members of the care team are involved, and how one's own actions would affect care delivery. ${ }^{15}$ This type of situational awareness is described in this passage from Practice 2, a site with a type-2 layout in which BHC and PCCs were clustered into small pods:

This pod had a pharmacist working there, a medical assistant, etc. It is tight. They do talk with each other when they come outside of the examination room. They do this regularly. They check in with each other to see where each is with working with the patients, and they get advice from each other. (Field Notes, Practice 2)

Close proximity also lends itself to visibility and "bumpability" (ie, the increased chance of physically encountering one another based on intention or chance) between BHCs and PCCs. Key informants from both type- 1 and type- 2 practices indicated that provider visibility and proximity had important implications for interprofessional interactions, as captured in the following excerpt:

If a primary care provider has to do more than this [gesture indicating simple or limited effort/motion] to be able to see where you are, they are not going to use you... I think the availability of a $\mathrm{BHC}$ is critical... So that immediate availability of consultation, going into the examination room on demand, talk- ing with a provider face to face immediately following, such that we're integrating the primary care and the behavioral health treatment plan into one in real time. (Interview with $\mathrm{BHC}$, Practice 2)

Although various strategies were employed across practices to bridge the distance between professionals, such as the use of cell phones, radios, and instant messaging in the electronic health record (EHR), key informants noted that these workarounds were no substitute for locating staff in close physical proximity.

Clinicians in practices with spatial arrangements that separated them from their colleagues (ie, type-1 layout) sometimes reported that they wished to be in closer proximity to other professionals. This was particularly noticeable when BHCs and PCCs had been working in close proximity and this was changed. For example, 1 PCC in Practice 11 noted that when the BHCs' work area was moved away from his pod it became much harder to access them. In addition, BHCs and PCCs located on different floors may not know each other well. In Practice 1 , primary care services were offered on 2 floors, and behavioral health services were located on a third separate floor. Although some BHCs and PCCs in this practice had developed personal relationships, others did not know each other and engagement of these behavioral health clinicians was only observed to occur during times of patient crisis (eg, suicide, domestic abuse). A practice leader commented: "If we have medical on one floor and psychiatry on another floor, I think that that is going to always keep things sort of separate." (Interview with Practice Leader, Practice 1)

The frequency in which we visibly observed BHCs and PCCs coordinating patient care (ie, 2 professionals working in a parallel or back-andforth fashion to achieve a common patient care goal while delivering care $)^{16}$ varied significantly across the 2 layout types. BHCs and PCCs coordinated care in $41.5 \%(n=39)$ of the total observed patient encounters in practices with shared work areas (type-2 layout) compared with $11.7 \%$ ( $\mathrm{n}=$ 14) of the total observed visits in practices with BHCs and PCCs in separate spaces (type-1 layout, $P<.05)$. 


\section{Private Work Space}

Shared workspace in some integrated practices came with a loss of privacy, and practices addressed this in a number of ways. In both type- 1 and type- 2 practice layouts, clinicians needed space to have private conversations, complete quiet and focused work, meet privately in groups to discuss patient care, and to deliver direct patient care. Practices designed new clinical rooms or repurposed existing clinical examination space into more conventional, "demedicalized" spaces. Such rooms were not scheduled as an examination room might be, but could be used on an as-needed basis, as shown in the following example:

This is a large practice that had been divided into smaller "clinics" in the same building. Each of the teams (eg, PCCs, case managers, medical assistants, schedulers) shared a pod in the practice, with the BHCs being in close proximity to the 2 pods they worked with. There is minimal privacy in these pods. Each team had 2 examination rooms and a talking room. A talking room is typically carpeted with a desk, computer, and a few chairs. We see these talking rooms being used for private consults and quiet work. At other times, clinicians use these rooms for overflow examination space or therapy space. (Field Notes, Practice 3)

Although Practice 3 did not have problems with privacy as it related to patient encounters, they reported problems trying to accomplish focused work in the common, open office space. Other practices reported similar challenges. To address this challenge, practices might have rooms available for additional medical examination purposes, consults, and therapy, and for work that needed quiet and privacy. This allowed practices to balance the need for individual and team work space and ameliorate professionals' feelings of overexposure by the increased visibility of their work.

\section{Renovating and Building for Integrated Care}

When a practice moves toward an integrated approach, they often bring new professionals into an existing practice to provide expanded services. Several approaches were used to modify existing phys- ical space to accommodate new team members. Strategies include reappropriating functional space, moving new clinicians into areas of high clinical workflow, and locating clinicians into shared offices. For example, in Practice 13 a small storage closet located in the midst of a busy primary care wing was repurposed for the BHC to see patients:

As we're walking upstairs the managing associate comments that the integrated care has worked because of changing physical locations for people... the manager later shows us that they are working to build a new $\mathrm{BH}$ [behavioral health] office in the family medicine office by converting an old storage closet. (Field Notes, Practice 13)

This room, although small and not shared, was directly in the workflow pathway for the primary care team, making the $\mathrm{BHC}$ in close proximity and easily accessible. The rationale and benefit of this type of space utilization is demonstrated by this explanation from the $\mathrm{BHC}$ at Practice 16:

I have deliberately positioned myself, my workspace, so that the providers can access me as easily as possible. And so that when they walk out of the room to grab their prescription pad they are like, 'Oh, the BHC! Yes, I also want this patient to come see you. Can you come in after me and talk to them about smoking cessation?' When I came to this practice the leadership said, 'We're going to give you an office. It'll be quiet for you...'; But, I do not want an office. I want to see patients in the examination room and I want to sit next to you. (Interview with BHC, Practice 16)

A strategy used by other BHCs was to carry their laptops with them in the hallway to be positioned in the midst of practice workflow (Practice 2). Other practices overcame distance between office locations by having BHCs and PCCs "hang out" in shared workspace, such as around the medical assistants' work stations.

Whether building new facilities or remodeling, we used study findings to work with an architect specializing in designing health care space to design 
2 layouts practice leaders might consider using when integrating physical and behavioral care teams. Figure 3 is an example floor plan that incorporates a central pod arrangement that allows for members of a care team to sit in close proximity to one another in an open space. Flexible rooms are available for quiet work, private consultations, individual or group counseling, and overflow examination space. The design depicted in Figure 3 could be beneficial for practices with smaller rooms, who might be able to make some modifications to an existing medical assistant station and add clinicians and other care team members to that space. Figure 4 differs in that team members share a central shared workspace in a closed office. This arrangement allows for more privacy among the staff and providers, but diminishes the visibility and "bumpability" among clinicians and support staff that is afforded by the design depicted in Figure 3. Figure 4 could be beneficial for practices with larger rooms that can be used to accommodate an entire care team.

\section{Discussion}

We assessed spatial designs and behaviors among professionals delivering integrated care in 19 practices located across the United States. Two spatial layouts emerged from our analysis, and were distinguished by the amount of separation between
PCCs and BHCs. Close proximity of BHCs and PCCs promoted situational awareness, "bumpability," and on-the-fly communication among professionals. In addition, clinicians working in closer proximity engaged in significantly more face-toface interactions to coordinating patient care than clinicians separated by distance. Our findings show that when designing or reconfiguring space for delivery of integrated care, practices must strike a balance between professional proximity and adequate private working space, given that private space was need for focused work and consults with patients or clinical team members.

Meaning is encoded within physical space..$^{2,17,18}$ By asserting control over a space and changing it (eg, from private to shared offices), individuals and groups can have a profound effect on the people they work with as well as the work that they do together. A common task of health care facilities is to deliver efficient, high-quality, patient-centered clinical care, and changes in the layout of physical spaces may facilitate or hinder accomplishment of these goals. Although studies describe the role that physical space plays in shaping interprofessional interactions, ${ }^{1-4}$ our study is one of the first to explore variations in physical layout, professional proximity, and care delivery patterns in a diverse spectrum of practices integrating behavioral health and primary care. Our findings are aligned with and

Figure 3. Recommended floor plan example, open pod arrangement. BHC, behavioral health clinician; Case Mgr, case manager; MA, medical assistant; PCC, primary care clinician; RN, registered nurse; Sched, Scheduler.

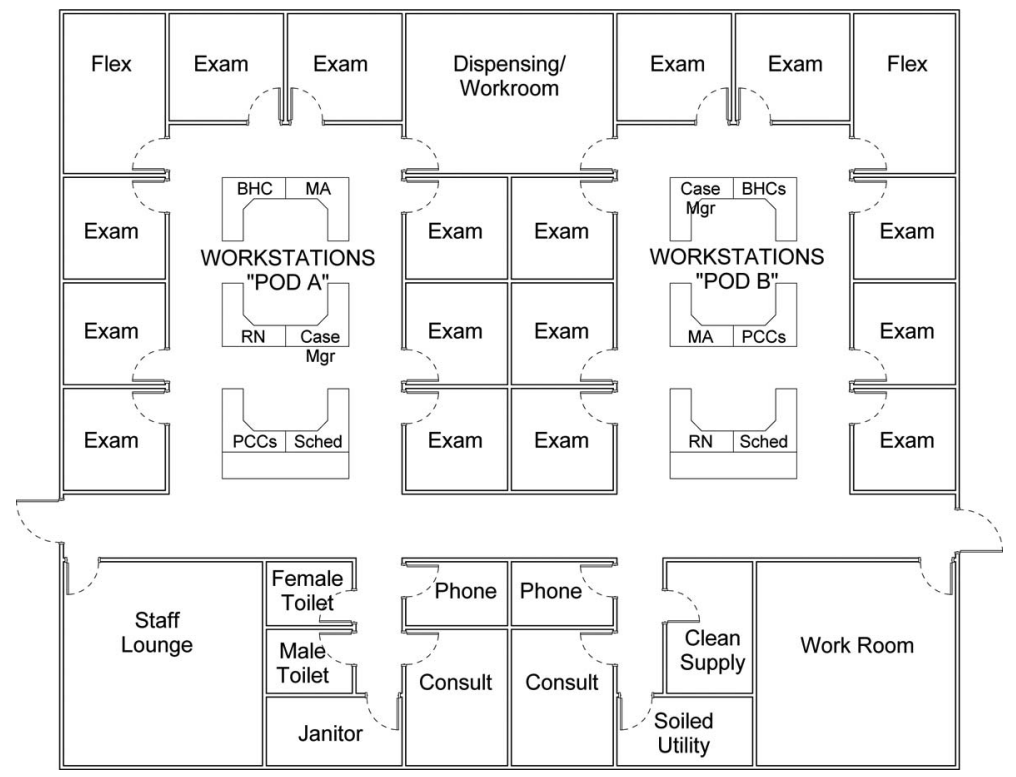


Figure 4. Recommended floor plan example, closed pod arrangement. BHC, behavioral health clinician; Case Mgr, case manager; EVS, environmental services; MA, medical assistant; PCC, primary care clinician; RN, registered nurse; Sched, Scheduler.

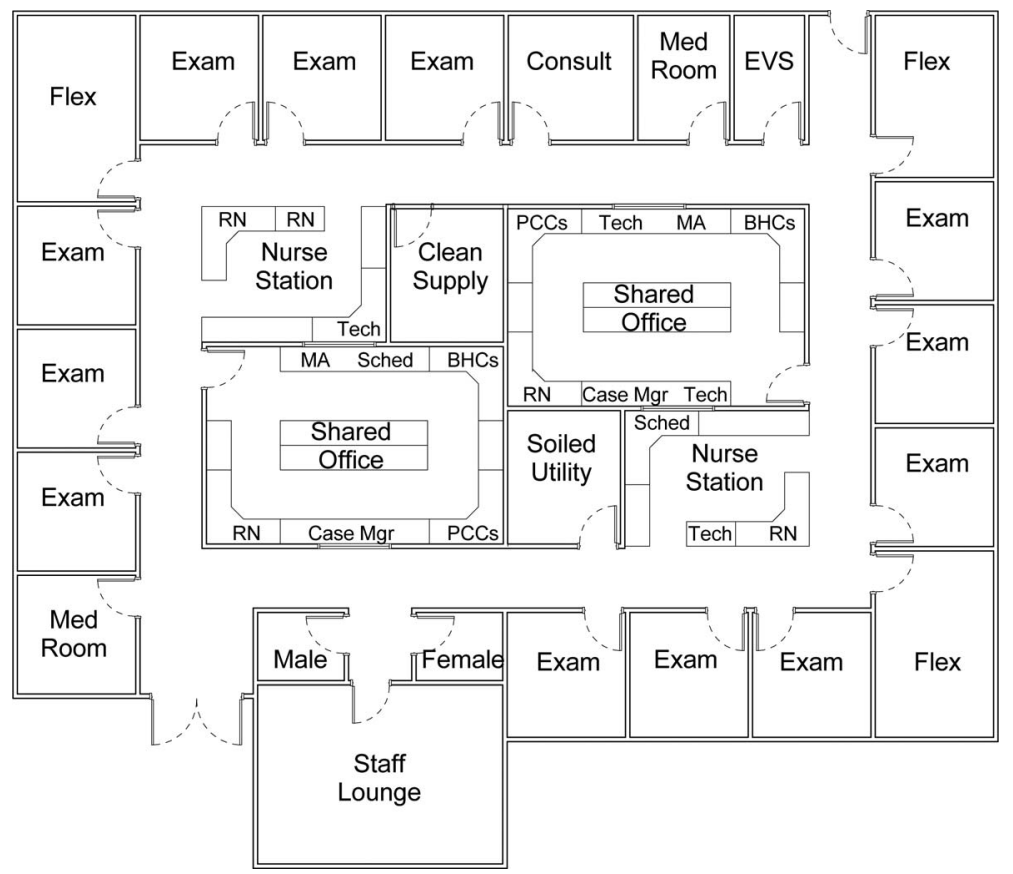

contribute to a small body of research suggesting that professionals working in close proximity experience greater ease in developing working relationships. $^{9-11,19,20}$ Study results reaffirm the importance of developing multi-purpose space, which offer places for both independent and team-based work, where employees can select the type of space best suited to the activities at hand. ${ }^{1}$

Many practices that aspire to provide integrated care do not have the luxury of creating new facilities; they have to make do with available space. Renovations can be costly, and the expenditures needed to renovate or build new space can be a large part of start-up expenses when transitioning to an integrated approach. ${ }^{21} \mathrm{Al}-$ though practices may struggle with a space that does not fully accommodate the new needs an integrated team, our findings show how these challenges can be ameliorated by a number of lower-cost strategies, including arranging more compact work areas that allow clinicians easy access to one another and close proximity of patient rooms to team workspace; and designating or repurposing flexible spaces outside of clinical examination rooms and workstations to provide for examination room overflow, quiet focused work, and private provider consultations.
There are a few important limitations in this work. First, much of the qualitative data we analyzed came from observations and interviews during site visits. Data reflect what was happening in the practice during these time periods and could be influenced by factors such as clinical team member absences. Second, we were unable to observe 2 of the practices first hand; we relied on other data sources (ie, online implementation diaries, interviews) to understand how space was used in these practices. We did not, therefore, include these practices in our counts of coordination. Third, what is observed during site visits can be shaped by the preconceptions of research team members. We mitigated this bias by having multiple team members collect and analyze study data, and by triangulating multiple data sources. ${ }^{22,23}$ Finally, our counts of coordinating behaviors were based on what we could see professionals doing (ie, working together face to face) and may not fully capture very subtle and less-visible mechanisms for coordinating delivery of integrated care, such as via an EHR. Results of the statistical comparison suggest that examining the relationships between space arrangements and interprofessional behaviors (eg, coordination) as well as other outcomes (eg, care quality, patient experience) may be worth additional study. 


\section{Conclusion}

Physical space can constrain or foster the integration of primary care and behavioral health services for patients. Clinical workflows and technologies, such as walkie-talkies or other communication devices, cannot entirely bridge spatial divides. Health care organizations committed to integrating care must adapt their current spatial arrangements or create new facility designs that support integration. In this study, a variety of enabling design features were utilized by a diverse range of practices working to integrate their primary and behavioral health care. Data from these integrated care environments provide practical information that clinicians, researchers, and health care administrators can use to design clinical spaces for integrated care that meets the needs of real-world clinics across the country.

The authors are grateful to the participating practices and their patients. The authors thank Leah Baruch, MD for her assistance with data collection on the IWS study and David Cameron, BA for his assistance with data analysis. The authors thank Bijal Balasubramanian, MBBS, PhD; Larry Green, MD; Frank deGruy III, MD; Sheldon Levy, PsyD; and Maribel Cifuentes, $\mathrm{RN}$, BSN for providing helpful comments during the preparation of this manuscript.

\section{References}

1. Boutellier R, Ullman F, Schreiber J, Naef R. Impact of office layout on communication in a science-driven business. R\&D Management 2008;38:372-91.

2. Hiller B, Hanson J. The Social Logic of Space. Cambridge: Cambridge University Press. 1984.

3. Keller AM, Joseph A, Taylor E, Quan X, Unruh C. Promising practices in safety-net clinic design: An overview. California HealthCare Foundation, 2011.

4. Brennan A, Chugh JS, Kline T. Traditional versus open office design: A longitudinal field study. Environment and behavior. 2002 2002;34:279-99.

5. Gulwadi GB, Joseph A, Keller AB. Exploring the impact of the physical environment on patient outcomes in ambulatory care settings. HERD 2009;2: 21-41.

6. Ulrich RS, Zimring C, Zhu X, et al. A review of the research literature on evidence-based healthcare design. HERD 2008;1:61-125.

7. Quan X, Joseph A, Keller A, Taylor E. Designing safety-net clinics for innovative care delivery models. California HealthCare Foundation, 2011.

8. Shepley M, Pasha S. Design research and behavioral health facilities. The Center for Health Design, 2013.
9. Lawn S, Lloyd A, King A, Sweet L, Gum L. Integration of primary health services: Being put together does not mean they will work together. BMC Res Notes 2014;7:66.

10. Oandasan IF, Gotlib Conn L, et al. The impact of space and time on interprofessional teamwork in Canadian primary health care settings: implications for health care reform. Primary Health Care Research \& Development 2009;10:151.

11. Calkins LE, Michelson IR, Corso AS. Provider proximity as a predictor of referral rate and success. Psychol Serv 2013;10:395-400.

12. Davis M, Balasubramanian BA, Waller E, Miller BF, Green LA, Cohen DJ. Integrating behavioral and physical health care in the real world: Early lessons from advancing care together. J Am Board Fam Med 2013;26:588-602.

13. Cohen DJ, Balasubramanian BA, Davis M, et al. Understanding care integration from the ground up: five organizing constructs that shape integrated practices. J Am Board Fam Med 2015;28:S7-S20.

14. Cohen DJ, Leviton LC, Isaacson N, Tallia AF, Crabtree BF. Online diaries for qualitative evaluation: Gaining real-time insights. Am J Eval 2006;27: 163-84.

15. Endsley MR. Toward a theory of situation awareness in dynamic systems. Human factors. J Human Factors Ergonomics Soc 1995;37:32-64.

16. Cohen DJ, Davis M, Balsubramanian BA, et al. Integrating behavioral health and primary care: consulting, coordinating and collaborating among professionals. J Am Board Fam Med 2015;28:S21-S31.

17. Foucault M, Miskowiec J. Of other spaces. Diacritics 1986;16:22-7.

18. Thrift N. Spatial formations. Thousand Oaks, CA: Sage, 1996.

19. Festinger L, Back KW, Schachter S. Social pressures in informal groups: A study of human factors in housing. Stanford University Press, 1950.

20. Morgan S, Pullon S, McKinlay E. Observation of interprofessional collaborative practice in primary care teams: An integrative literature review. International Journal of Nursing Studies. 2015;52: 1217-30.

21. Wallace NT, Cohen DJ, Gunn R, et al. Start-up and ongoing practice expenses of behavioral health and primary care integration interventions in the $\mathrm{Ad}$ vancing Care Together (ACT) program. J Am Board Fam Med 2015;28:S86-S97.

22. Cohen DJ, Crabtree BF. Evaluative criteria for qualitative research in health care: Controversies and recommendations. Ann Fam Med 2008;6:331-9.

23. Whittemore R, Chase SK, Mandle CL. Validity in qualitative research. Qual Health Res. 2001;11: 522-37. 